\title{
ANALYSIS OF PRESSURE VARIATION OF FLUID IN BOUNDED CIRCULAR RESERVOIRS UNDER THE CONSTANT PRESSURE OUTER BOUNDARY CONDITION
}

\author{
I. D. Erhunmwun ${ }^{1, *}$ and J. A. Akpobi² \\ 1, 2 Department of Production Engineering, University of Benin, Benin City, Edo State. NiGERIA. \\ E-mail addresses: 1 iredia.erhunmwun@uniben.edu, 2 john.akpobi@uniben.edu
}

\begin{abstract}
In this work, we have investigated the well pressure distribution in a bounded circular reservoir under the condition of constant pressure outer boundaries. The diffusivity equation was used in the analysis. The finite element technique, using Lagrange quadratic shape elements was employed to carry out the analysis over the cross-section of the reservoir which involves discretizing the domain into finite element, analysing these finite element, assembling the results from the analysis of the analysed finite element, imposing the boundary conditions and finally, getting the results that represent the entire domain. The results obtained where shown in a log log plot (dimensionless pressure against dimensionless time) for dimensionless radii of 1 to 1,000,000 in log cycles. It was shown that the relationship between dimensionless pressure and dimensionless time was linear whose slope was zero. The result obtained at the wellbore was compared with the results obtained by Van Everdigen and Hurst. It was shown that there was a strong positive correlation between the results. The result obtained from the analysis also shows the pressure variation outside wellbore of the same reservoir. It is important to note that solutions from existing literature only state the pressure at the wellbore at a particular time but this work predicts the pressure variation in the entire reservoir from the wellbore to the external boundary at the same time.
\end{abstract}

Keywords: Reservoir, Constant Terminal Rate, Dimensionless Variables, Diffusivity Equation, Wellbore And Weak Formulation.

Nomenclature
English Letters
$B \quad$ Formation volume factor, $\mathrm{RB} / \mathrm{STB}$
$c \quad$ Compressibility, $\mathrm{psia}^{-1}$
$h \quad$ Thickness, ft
$K$ Stiffness matrix
$M$ Mass matrix
$n \quad$ Number of elements
$P \quad$ Pressure, psi
$P_{D}$ Dimensionless pressure
$\dot{P_{D}}$ Dimensionless pressure rate

\section{INTRODUCTION}

There are several methods of evaluating the reservoir parameters [1]. It was shown that solutions to differential equations describing flow in petroleum reservoir for given initial and boundary conditions can be expressed compactly using dimensionless variables and parameters. Several of these solutions are important in reservoir engineering applications [2-7]. Transient
$P_{i} \quad$ Initial reservoir pressure, psi

$Q$ Terminal flow rate

$q$ Volumetric flow rate, STB/D

$r$ Radius, $\mathrm{ft}$

$r_{D}$ Dimensionless radius

$r_{e}$ External radius, $\mathrm{ft}$

$r_{e D}$ Dimensionless externalradius

$r_{w}$ Wellbore radius, $\mathrm{ft}$

$s$ Time step, hr

$t$ Time, hr

$t_{D}$ Dimensionless time

\author{
$w$ Weight function \\ $\forall$ For all \\ Greek letters \\ $\Delta t$ Time increment, hr \\ $\alpha$ Family of approximation \\ $\phi$ Porosity, fraction \\ $k$ Permeability, md \\ $\mu$ Viscosity, cp \\ $\pi \mathrm{Pi}$ \\ $\psi$ Interpolation function
}

pressure response for a well producing from a finite reservoir of circular, square, and rectangular drainage shapes has been studied by [2, 8-14] among others. Everdingen And Hurst [2] presented the solution to the diffusivity equation in eq. (8) in the form of infinite series of exponential terms and Bessel functions. The authors evaluated this series for several values of $r_{e D}$ over a wide range of values for $t_{D}$. Chatas [15] and Well

\footnotetext{
* Corresponding author, tel: +234-807- $072-8898$
} 
[16] conveniently tabulated these solutions for the following two cases: Infinite-acting reservoir and Finiteradial reservoir.

Mishra and Ramey [17] presented a build-up derivative type curve for a well with storage and skin, and producing from the centre of a closed, circular reservoir. Their type-curve applies for large producing times. The work by [18] presents drawdown and build-up pressure derivative type-curves for a well producing at a constant rate from the centre of a finite, circular reservoir. The outer boundary may be closed, or at a constant pressure. The differences between the responses for a well in a closed, circular reservoir (fully developed field), and a well in a circular reservoir with a constant-pressure outer boundary (active edge water drive system, or developed five-spot fluid-injection pattern) were discussed. Design relations were developed to estimate the time period which corresponds to infinite-acting radial flow, or to a semi-log straight line on a pressure vs. logarithm of time graph. Producing time effects on buildup responses were studied using the slope of a dimensionless build-up graph proposed in [19].

In all the literatures reviewed so far, the researchers focused on predicting the wellbore pressure $[2,15,16]$, etc. Sometimes, it is important to know the pressure history outside the reservoir is scarce in the literature. This study therefore seeks to look at the reservoir pressure both within and outside the wellbore of a bounded circular reservoir.

\section{THEORY}

The law of conservation of mass, Darcy's law and the equation of state has been combined to obtain the following partial differential equation:

$$
\frac{\partial^{2} P}{\partial r^{2}}+\frac{1}{r} \frac{\partial P}{\partial r}=\frac{\emptyset \mu c}{0.000264 k} \frac{\partial P}{\partial t}
$$

with the assumptions that compressibility, $c$ is small and independent of pressure, $\mathrm{P}$; permeability, $\mathrm{k}$, is constant and isotropic; viscosity, , $\mu$ is independent of pressure; porosity, $\varnothing$ is constant; and that certain terms in the basic differential equation (involving pressure gradients squared) are negligible. This equation is called the diffusivity equation and the term $\frac{\emptyset \mu c}{0.000264 k}$ is the inverse of the diffusivity constant,. $\mu$

In this work, the diffusivity equation was analysed for bounded circular reservoirs, the case in which the well is assumed to be located in the centre of a cylindrical reservoir under the condition of constant external boundary.

\section{GOVERNING EQUATION}

$$
\frac{\partial^{2} P}{\partial r^{2}}+\frac{1}{r} \frac{\partial P}{\partial r}=\frac{\emptyset \mu c}{0.000264 k} \frac{\partial P}{\partial t}
$$

Initial and boundary conditions:

$$
\begin{aligned}
& \text { i. } \quad P=P_{i} \text { at } \mathrm{t}=0 \forall r \\
& \text { ii. } \quad\left(r \frac{\partial P}{\partial r}\right)_{r_{w}}=\frac{q^{B} \mu}{2 \pi k h} \text { for }>.0 \\
& \text { iii. }\left(r \frac{\partial P}{\partial r}\right)_{r_{w}}=0 \forall t
\end{aligned}
$$

The above equations incorporate physical parameters such as permeability, and it would be futile to solve this problem for a particular combination of values for these parameters. Dimensionless variables are designed to eliminate the physical parameters that affect quantitatively, but not qualitatively, the reservoir response. The above equations are in Darcy units, and the dimensionless terms will render the system of units employed irrelevant. For this line source model, 3 dimensionless variables are required. In US Oilfield units, distance, time and pressure are replaced as follows:

Dimensionless time:

$$
t_{D}=\frac{0.0002637 k t}{\emptyset \mu c r_{w}^{2}}
$$

Dimensionless distance:

$$
r_{D}=\frac{r}{r_{w}}
$$

Dimensionless pressure:

$$
P_{D}=\frac{k h}{141.2_{q} B_{\mu}}\left(P_{1}-P\right)
$$

By defining dimensionless variables this way, it is possible to create an analytical model of the well and reservoir, or theoretical 'type-curve', that provides a 'global' description of the pressure response that is independent of the flow rate or actual values of the well and reservoir parameters.

Eq.1 can be transformed by substituting the following dimensionless variables in Eqs. 5-7 into eq. 1 and this becomes:

$$
\frac{\partial^{2} P_{D}}{\partial r_{D}{ }^{2}}+\frac{1}{r_{D}} \frac{\partial P_{D}}{\partial r_{D}}=\frac{\partial P_{D}}{\partial t_{D}}
$$

and the initial and boundary conditions becomes:

1. Dimensionless initial condition (uniform pressure in the reservoir):

$$
P_{D}\left(r_{D}, t_{D}=0\right)
$$

2. Dimensionless inner boundary condition (constant rate at the wellbore):

$$
\frac{\partial P_{D}}{\partial r_{D}}\left(1, t_{D}\right)=-1
$$

3. Dimensionless Outer Boundary Conditions: Constant pressure outer boundary

$$
P_{D}=\left(r_{e D}, t_{D}\right)=0
$$

Eq. 8 can also be written in a condensed form as:

$$
\frac{1}{r_{D}} \frac{\partial}{\partial r_{D}}\left(r_{D} \frac{\partial P_{D}}{\partial r_{D}}\right)=\frac{\partial P_{D}}{\partial t_{D}}
$$




\section{FINITE ELEMENT FORMULATION}

\subsection{Weak Formulation}

In the development of the weak form, we assumed a quadratic element mesh and placed it over the domain and apply the following steps:

From eq. 12, we have:

$$
\frac{\partial P_{D}}{\partial t_{D}}-\frac{1}{r_{D}} \frac{\partial}{\partial r_{D}}\left(r_{D} \frac{\partial P_{D}}{\partial r_{D}}\right)=0
$$

Multiply eq. 13 by the weight $w$ function and integrate the final equation over the domain.

$$
\int_{v} w\left[\frac{\partial P_{D}}{\partial t_{D}}-\frac{1}{r_{D}} \frac{\partial}{\partial r_{D}}\left(r_{D} \frac{\partial P_{D}}{\partial r_{D}}\right)\right] d v=0
$$

Eq. 14 becomes,

$$
\int_{0}^{1} \int_{0}^{2 \pi r} \int_{r_{D A}}^{D B} w\left[\frac{\partial P_{D}}{\partial t_{D}}-\frac{1}{r_{D}} \frac{\partial}{\partial r_{D}}\left(r_{D} \frac{\partial P_{D}}{\partial r_{D}}\right)\right] r_{D} d r_{D} d \theta d z=0
$$

Integrating eq. 15 with respect to $z$, then, $\theta$ over the limits, we have:

$$
\int_{r_{D A}}^{r_{D B}} w\left[\frac{\partial P_{D}}{\partial t_{D}}+\frac{1}{r_{D}} \frac{\partial}{\partial r_{D}}\left(r_{D} \frac{\partial P_{D}}{\partial r_{D}}\right)\right] r_{D} d r_{D}=0
$$

Eq. 16 can be exploded into:

$$
\int_{r_{D A}}^{r_{D B}} w \frac{\partial P_{D}}{\partial t_{D}} r_{D} d r_{D}-\int_{r_{D A}}^{r_{D B}} w \frac{\partial}{\partial r_{D}}\left(r_{D} \frac{\partial P_{D}}{\partial r_{D}}\right) d r_{D}=0
$$

Integrating eq. 17 by part, we have:

$$
\begin{gathered}
\int_{r_{D A}}^{r_{D B}} r_{D} \frac{\partial w}{\partial r_{D}} \frac{\partial P_{D}}{\partial r_{D}} d r_{D}-w\left[r_{D} \frac{\partial P_{D}}{\partial r_{D}}\right]_{r_{D A}}^{r_{D B}}+\int_{r_{D A}}^{r_{D B}} r_{D} w \frac{\partial P_{D}}{\partial t_{D}} d r_{D} \\
=0
\end{gathered}
$$

Grouping eq. 18 into linear and bilinear components, we have:

$$
\begin{gathered}
\int_{r_{D A}}^{r_{D B}} r_{D} \frac{\partial w}{\partial r_{D}} \frac{\partial P_{D}}{\partial r_{D}} d r_{D}+\int_{r_{D A}}^{r_{D B}} r_{D} w \frac{\partial P_{D}}{\partial t_{D}} d r_{D}-w\left[r_{D} \frac{\partial P_{D}}{\partial r_{D}}\right]_{r_{D A}}^{r_{D B}} \\
=0 \\
\int_{r_{D A}}^{r_{D B}} r_{D} \frac{\partial w}{\partial r_{D}} \frac{\partial P_{D}}{\partial r_{D}} d r_{D}+\int_{r_{D A}}^{r_{D B}} r_{D} w \frac{\partial P_{D}}{\partial t_{D}} d r_{D}-w Q_{A}-w Q_{B} \\
=0
\end{gathered}
$$

Where $Q=r_{D} \frac{\partial P_{D}}{\partial r_{D}}$

\section{INTERPOLATION FUNCTION}

The weak form in eq. 20 requires that the approximation chosen for $P_{D}$ should be at least quadratic in $r_{D}$ so that there are no terms in eq. 20 that are identically zero. Since the primary variable is simply the function itself, the Lagrange family of interpolation functions is admissible. We proposed that $P_{D}$ is the approximation over a typical finite element domain by the expression:

$$
P_{D}\left(r_{D}, t_{D}\right)=\sum_{j-1}^{n} P_{D j}\left(t_{D}\right) \psi_{j}^{e}\left(r_{D}\right) \text { and } w=\psi_{j}^{e}\left(r_{D}\right)
$$

Substituting eq. 21 into eq. 20, we have:

$$
\begin{gathered}
\int_{r_{D A}}^{r_{D B}} r_{D} \frac{d \psi_{j}^{e}}{d r_{D}} \frac{\partial}{d r_{D}} \sum_{j-1}^{n} P_{D j}\left(t_{D}\right) \psi_{j}^{e}\left(r_{D}\right) d r_{D} \\
+\int_{r_{D A}}^{r_{D B}} r_{D} \psi_{j}^{e} \frac{d}{d t_{D}} \sum_{j-1}^{n} P_{D j}\left(t_{D}\right) \psi_{j}^{e}\left(r_{D}\right) d r_{D} \\
-Q_{i}^{e}=0
\end{gathered}
$$

Factor out $\sum_{j-1}^{n} P_{D j}$

$$
\begin{gathered}
\sum_{j-1}^{n} P_{D j}+\int_{r_{D A}}^{r_{D B}} r_{D} \frac{d \psi_{i}^{e}}{d r_{D}} \frac{d \psi_{j}^{e}}{d r_{D}} d r_{D}+\sum_{j-1}^{n} \dot{P}_{D j} \int_{r_{D A}}^{r_{D B}} r_{D} \psi_{i}^{e} \psi_{j}^{e} d r_{D} \\
-Q_{i}^{e}=0 \\
\text { where } \dot{P}_{D j}=\frac{d P_{D}}{d t_{D}}
\end{gathered}
$$

In matrix form we can represent the semi-discrete finite element model as thus,

$$
\left|K_{i j}^{e}\right|\left\{P_{D}\right\}+\left|M_{i j}^{e}\right|\left\{\dot{P}_{D j}\right\}=\left\{Q_{i}^{e}\right\}
$$

Where

$$
\begin{aligned}
K_{i j}^{e} & =\int_{r_{D A}}^{r_{D B}} r_{D} \frac{d \psi_{i}^{e}}{d r_{D}} \frac{d \psi_{j}^{e}}{d r_{D}} d r_{D} \\
M_{i j}^{e} & =\int_{r_{D A}}^{r_{D B}} r_{D} \psi_{i}^{e} \psi_{j}^{e} d r_{D}
\end{aligned}
$$

Using Quadratic Lagrange Interpolation functions for a quadratic element:

$$
\begin{array}{r}
\psi_{1}(r)=\frac{1}{h^{2}}\left(h+r_{A}-r\right)\left(h-2 r+2 r_{A}\right) \\
\psi_{2}(r)=\frac{4}{h^{2}}\left(r-r_{A}\right)\left(h+r_{A}-r\right) \\
\psi_{3}(r)=\frac{-1}{h^{2}}\left(r-r_{A}\right)\left(h-2 r+2 r_{A}\right)
\end{array}
$$

The coefficient matrix can be easily derived by substituting the Lagrange interpolation functions into eq. 25 respectively. The matrices are shown below:

$\left[K^{e}\right]$

$=\frac{1}{6 h}\left[\begin{array}{ccc}3 h+14 r_{A} & -\left(4 h+16 r_{A}\right) & h+2 r_{A} \\ -\left(4 h+16 r_{A}\right) & 16 h+32 r_{A} & -\left(12 h+16 r_{A}\right) \\ h+2 r_{A} & -\left(12 h+16 r_{A}\right) & 11 h+14 r_{A}\end{array}\right]$

Also, the mass matrices can be easily derived by substituting the Lagrange interpolation functions into eq. 26 respectively. The matrices are shown below:

$$
\left[M^{e}\right]=\frac{1}{60}\left[\begin{array}{ccc}
h+8 r_{A} & 4 r_{A} & -h-2 r_{A} \\
4 r_{A} & 16 h+32 r_{A} & 4 h+4 r_{A} \\
-h-2 r_{A} & 4 h+4 r_{A} & 7 h+8 r_{A}
\end{array}\right]
$$

Using four quadratic elements,

$$
r_{A}=r_{w}+(n-1) h
$$

In this analysis, we have withheld the computational details of the shape assembly of the finite element 
analysis (FEA) used. However, the authors would be glad to interact with researchers who may want to refer to the computational mathematics involved.

\section{TIME APPROXIMATION}

For a given time step s, eq. 24 will be written as

$$
\left|K_{i j}^{e}\right|\left\{P_{D}\right\}+\left|M_{i j}^{e}\right|\left\{\dot{P}_{D j}\right\}=\left\{Q_{i}^{e}\right\}
$$

For the next time step s+1, eq. 24 becomes

$$
\left|K_{i j}^{e}\right|\left\{P_{D}\right\}_{s+1}+\left|M_{i j}^{e}\right|\left\{\dot{P}_{D j}\right\}_{s+1}=\left\{Q_{i}^{e}\right\}_{s+1}
$$

Multiply eq.33 by $(1-\alpha)$ and eq. 34 by $\alpha$, then we add the two resulting equations,

$$
\begin{aligned}
{\left[M_{i j}^{e}\right]\left[(1-a)\left\{\dot{P}_{D j}\right\}_{s}+a\left\{\dot{P}_{D j}\right\}_{s+1}\right] } \\
+\left[K_{i j}^{e}\right]\left[(1-a)\left\{P_{D j}\right\}_{s}+a\left\{P_{D j}\right\}_{s+1}\right] \\
=(1-a)\left\{Q_{i}^{e}\right\}_{s}+\left\{Q_{i}^{e}\right\}_{s+1}
\end{aligned}
$$

The $\alpha$ family of interpolation for time consideration is given as:

$$
(1-a)\left\{\dot{P}_{D j}\right\}_{s}+a\left\{\dot{P}_{D j}\right\}_{s+1}=\frac{\left\{P_{D j}\right\}_{s+1}-\left\{P_{D j}\right\}_{s}}{\Delta t_{s+1}}
$$

Substitute eq.36 into eq.35 and using the CrankNicholson Scheme where $\alpha=1 / 2$,

$$
\begin{aligned}
{\left[\left[M_{i j}^{e}\right]+\frac{\Delta t_{s+1}}{2}\left[K_{i j}^{e}\right]\right]\left\{P_{D j}\right\}_{s+1} } \\
=\left[\left[M_{i j}^{e}\right]-\frac{\Delta t_{s+1}}{2}\left[K_{i j}^{e}\right]\right]\left\{P_{D j}\right\}_{s} \\
+\frac{\Delta t_{s+1}}{2}\left[\left\{Q_{i}^{e}\right\}_{S}+\left\{Q_{i}^{e}\right\}_{s+1}\right]
\end{aligned}
$$

From the initial condition given in eq. 9 for a constant terminal rate case, it implies that when $s=0$, all dimensionless pressure in the reservoir will be zero. Also, the flow rate was constant at the wellbore all through operation. This means that $\left[\left\{Q_{i}^{e}\right\}_{s}+\right.$ $\left.\left\{Q_{i}^{e}\right\}_{s+1}\right]$.Hence, eq. 37 becomes:

$$
\begin{aligned}
{\left[\left[M_{i j}^{e}\right]+\frac{\Delta t_{s+1}}{2}\left[K_{i j}^{e}\right]\right]\left\{P_{D j}\right\}_{1} } & \\
= & {\left[\left[M_{i j}^{e}\right]-\frac{\Delta t_{1}}{2}\left[K_{i j}^{e}\right]\left\{P_{D j}\right\}_{0}\right.} \\
& \left.+\Delta t_{1}\left\{\bar{Q}_{i}^{e}\right\}\right]
\end{aligned}
$$

Where $\bar{Q}_{i}^{e}=\frac{1}{2}\left(Q_{i}^{1}\right)_{s+1}+\frac{1}{2}\left(Q_{i}^{1}\right)_{s}$

$$
\begin{aligned}
\left\{P_{D j}\right\}_{1}=\left[\left[M_{i j}^{e}\right]+\right. & \left.\frac{\Delta t_{1}}{2}\left[K_{i j}^{e}\right]\right]^{-1}\left[\left[M_{i j}^{e}\right]-\frac{\Delta t_{1}}{2}\left[K_{i j}^{e}\right]\right]\left\{P_{D j}\right\}_{0} \\
& +\Delta t_{1}\left\{\bar{Q}_{i}^{e}\right\}
\end{aligned}
$$

\section{RESULTS AND DISCUSSION}

The steady state condition applies, after the transient period, to a well-draining a cell which has a completely open outer boundary. It is assumed that, for a constant rate of production, fluid withdrawal from the well will be exactly balanced by fluid entry across the outer boundary and therefore,

$$
\begin{gathered}
P=P_{e}=\text { Constant at } r \text { i. e. }, . P_{D}\left(r_{D} \rightarrow r_{e D}, t_{D}\right)=0 \text { and } \frac{\partial \mathrm{P}}{\partial \mathrm{t}} \\
=0 \forall r \text { and } t \forall
\end{gathered}
$$

This condition is appropriate when pressure is being maintained in the reservoir due to either natural water influx or artificially by the injection of some displacing fluid. The semi-steady state flow equations are frequently applied when the rate, and consequently the position of the no-flow boundary surrounding a well, is slowly varying functions of time. If the production rate of an individual well is changed, for instance, due to closure for repair or increasing the rate to obtain a more even fluid withdrawal pattern throughout the reservoir, there will be a brief period when transient flow conditions prevail followed by stabilized flow for the new distribution of individual well rates.

Thus, this solution of the diffusivity equation models radial flow of slightly compressible liquid in a homogeneous reservoir of uniform thickness; reservoir at uniform pressure before production; unchanging pressure at the outer boundary; and production at constant rate from a single well (centred in the reservoir) with wellbore radius.

The results obtained from this analysis were shown in the form of graphs of dimensionless pressure against dimensionless time. This was shown in Fig. 1. Fig. 1 is a log log plot of dimensionless pressure against dimensionless time. The graph shows for different dimensionless radii ranging between 1 and 1000000 in $\log$ cycles. It was seen from the graph that the dimensionless pressure history of the reservoir was not captured at the initial stage between the dimensionless time of zero and the respective dimensionless times in Fig. 1. This was due to the fact that, within these regions, the reservoir was at the infinite acting state. After these infinite acting period, it was observed that the dimensionless pressure increases and later becomes uniform became the withdrawn fluid has been completely replaced. This condition remains throughout the entire life of the reservoir presumably.

To test for the degree of accuracy of the results, a percentage error computation was done between the FEM and the results published by [2]. Table 1 shows the percentage error between the FEM solutions and the Van Everdigen and Hurst solutions to show the level of discrepancies between the two results. It was shown that there was a strong correlation between the two results. 


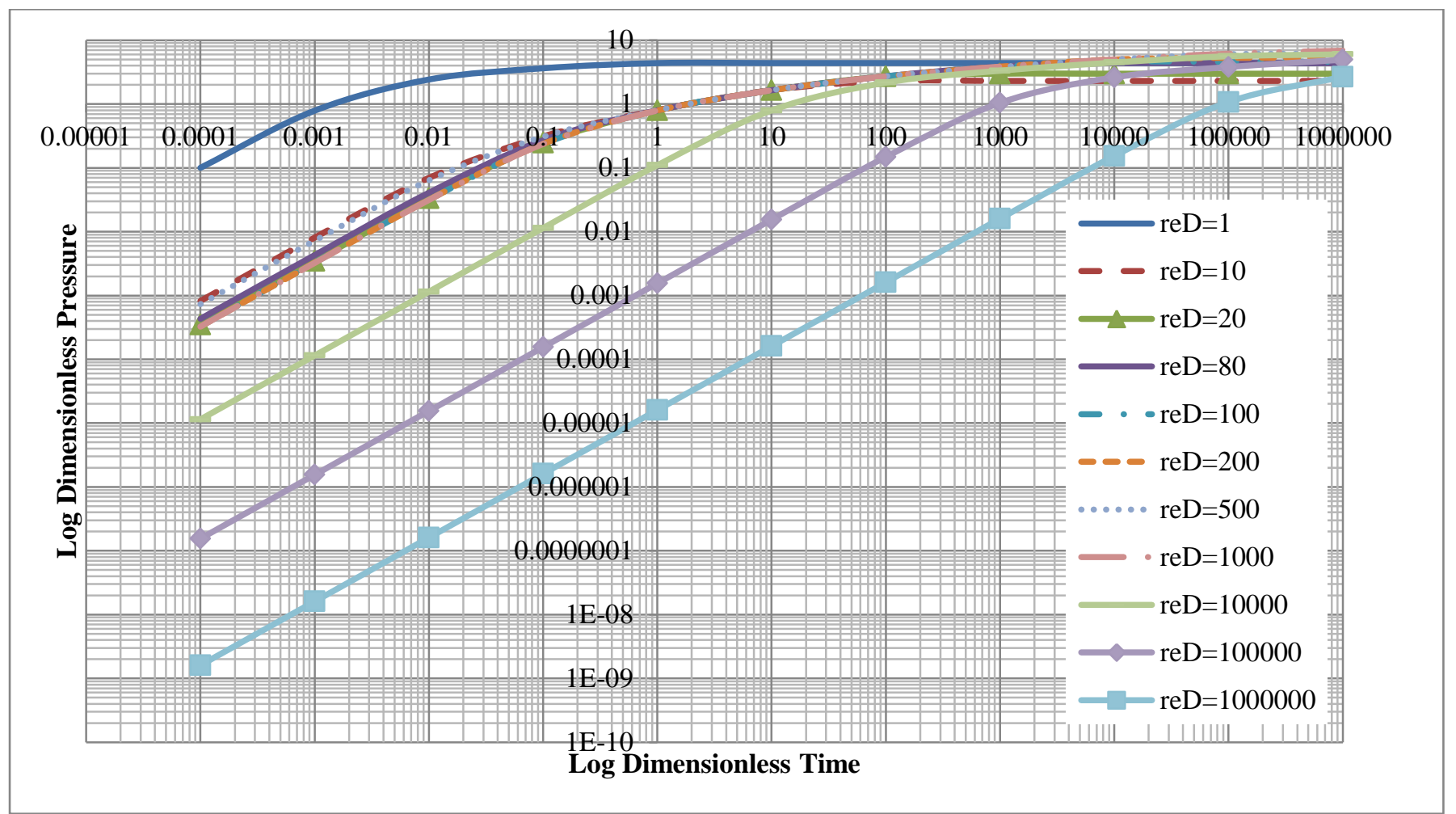

Fig. 1: Log-Log plot of PD against $t D$ for various $r_{D}$ in the infinite acting regime

Table 1: Percentage error of FEM and Van Everdigen

\begin{tabular}{|c|c|c|c|c|c|c|c|c|c|c|c|c|c|}
\hline \multicolumn{2}{|c|}{$r_{e D}=1.5, n=4$} & \multicolumn{2}{|c|}{$r_{e D}=2, n=4$} & \multicolumn{2}{|c|}{$r_{e D}=2.5, n=4$} & \multicolumn{2}{|c|}{$r_{e D}=3, n=4$} & \multicolumn{2}{|c|}{$r_{e D}=3.5, n=4$} & \multicolumn{2}{|c|}{$r_{e D}=4, n=4$} & \multicolumn{2}{|c|}{$r_{e D}=6, n=4$} \\
\hline$t_{D}$ & $\%$ error & $t_{D}$ & $\%$ error & $t_{D}$ & $\%$ error & $t_{D}$ & $\%$ error & $t_{D}$ & $\%$ error & $t_{D}$ & $\%$ error & $t_{D}$ & $\%$ error \\
\hline 0.05 & 0.4348 & 0.2 & 0.0000 & 0.3 & 0.0000 & 0.5 & 0.1621 & 0.5 & 0.4839 & 1 & 0.0000 & 4 & 0.2353 \\
\hline 0.055 & 0.4167 & 0.22 & 0.0000 & 0.35 & 0.0000 & 0.55 & 0.1563 & 0.6 & 0.4511 & 1.2 & 0.1167 & 4.5 & 0.1515 \\
\hline 0.06 & 0.0000 & 0.24 & 0.0000 & 0.4 & 0.0000 & 0.6 & 0.0000 & 0.7 & 0.4255 & 1.4 & 0.1105 & 5 & 0.1470 \\
\hline 0.07 & 0.0000 & 0.26 & 0.0000 & 0.45 & 0.0000 & 0.7 & 0.0000 & 0.8 & 0.2699 & 1.6 & 0.0000 & 5.5 & 0.1431 \\
\hline 0.08 & 0.0000 & 0.28 & 0.0000 & 0.5 & 0.0000 & 0.8 & 0.0000 & 0.9 & 0.3876 & 1.8 & 0.1014 & 6 & 0.1397 \\
\hline 0.09 & 1.0274 & 0.3 & 0.0000 & 0.55 & 0.0000 & 0.9 & 0.0000 & 1 & 0.2488 & 2 & 0.0000 & 6.5 & 0.1368 \\
\hline 0.1 & 0.0000 & 0.35 & 0.0000 & 0.6 & 0.0000 & 1 & 0.0000 & 1.2 & 0.2331 & 2.2 & 0.0951 & 7 & 0.1342 \\
\hline 0.12 & 0.0000 & 0.4 & 0.0000 & 0.7 & 0.0000 & 1.2 & 0.0000 & 1.4 & 0.1106 & 2.4 & 0.0000 & 7.5 & 0.1319 \\
\hline 0.14 & 0.0000 & 0.45 & 0.1748 & 0.8 & 0.1374 & 1.4 & 0.0000 & 1.6 & 0.1058 & 2.6 & 0.5425 & 8 & 0.1300 \\
\hline 0.16 & 0.0000 & 0.5 & 0.0000 & 0.9 & 0.1325 & 1.6 & 0.1079 & 1.8 & 0.1019 & 2.8 & 0.0000 & 8.5 & 0.1281 \\
\hline 0.18 & 0.0000 & 0.55 & 0.0000 & 1 & 0.0000 & 1.8 & 0.0000 & 2 & 0.0988 & 3 & 0.0000 & 9 & 0.1266 \\
\hline 0.2 & 0.0000 & 0.6 & 0.0000 & 1.2 & 0.1227 & 2 & 0.0000 & 2.2 & 0.1921 & 3.4 & 0.0000 & 10 & 0.1238 \\
\hline 0.22 & 0.0000 & 0.65 & 0.0000 & 1.4 & 0.0000 & 2.2 & 0.0000 & 2.4 & 0.0939 & 3.8 & 0.0000 & 12 & 0.1200 \\
\hline 0.24 & 0.0000 & 0.7 & 0.0000 & 1.6 & 0.0000 & 2.4 & 0.0000 & 2.6 & 0.0920 & 4.5 & 0.0000 & 14 & 0.1174 \\
\hline 0.26 & 0.0000 & 0.75 & 0.0000 & 1.8 & 0.0000 & 2.6 & 0.0000 & 2.8 & 0.0904 & 5 & 0.0000 & 16 & 0.0578 \\
\hline 0.28 & 0.0000 & 0.8 & 0.0000 & 2 & 0.0000 & 2.8 & 0.0000 & 3 & 0.0000 & 5.5 & 0.0000 & 18 & 0.1144 \\
\hline 0.3 & 0.0000 & 0.85 & 0.0000 & 2.2 & 0.0000 & 3 & 0.0000 & 3.5 & 0.0864 & 6 & 0.0755 & 20 & 0.1135 \\
\hline 0.35 & 0.0000 & 0.9 & 0.0000 & 2.4 & 0.0000 & 3.5 & 0.0000 & 4 & 0.0845 & 7 & 0.0742 & 22 & 0.1129 \\
\hline 0.4 & 0.0000 & 0.95 & 0.0000 & 2.6 & 0.0000 & 4 & 0.0000 & 5 & 0.0000 & 8 & 0.0000 & 24 & 0.1125 \\
\hline 0.45 & 0.0000 & 1 & 0.0000 & 2.8 & 0.0000 & 4.5 & 0.0000 & 6 & 0.0000 & 9 & 0.0000 & 26 & 0.1123 \\
\hline 0.5 & 0.0000 & 1.2 & 0.0000 & 3 & 0.0000 & 5 & 0.0000 & 7 & 0.0000 & 10 & 0.0000 & 28 & 0.0561 \\
\hline
\end{tabular}


Table 2: Dimensionless Pressure Distribution for $r_{e D}=1.5, n=4$ and $\Delta t=0.005$

\begin{tabular}{cccccccccc}
\hline $\mathrm{tD}$ & 1 & 1.0625 & 1.1250 & 1.1875 & 1.2500 & 1.3125 & 1.3750 & 1.4375 & 1.5000 \\
\hline 0.050 & 0.229 & 0.174 & 0.127 & 0.091 & 0.063 & 0.041 & 0.025 & 0.011 & 0.000 \\
0.055 & 0.241 & 0.183 & 0.137 & 0.099 & 0.070 & 0.046 & 0.028 & 0.013 & 0.000 \\
0.060 & 0.249 & 0.192 & 0.145 & 0.107 & 0.076 & 0.051 & 0.031 & 0.015 & 0.000 \\
0.070 & 0.266 & 0.209 & 0.160 & 0.121 & 0.087 & 0.060 & 0.037 & 0.018 & 0.000 \\
0.080 & 0.282 & 0.224 & 0.174 & 0.133 & 0.098 & 0.068 & 0.043 & 0.020 & 0.000 \\
0.090 & 0.295 & 0.237 & 0.187 & 0.144 & 0.107 & 0.075 & 0.048 & 0.023 & 0.000 \\
0.100 & 0.307 & 0.249 & 0.198 & 0.154 & 0.115 & 0.082 & 0.052 & 0.025 & 0.000 \\
0.120 & 0.328 & 0.269 & 0.216 & 0.170 & 0.129 & 0.092 & 0.059 & 0.029 & 0.000 \\
0.140 & 0.344 & 0.284 & 0.231 & 0.183 & 0.140 & 0.101 & 0.065 & 0.032 & 0.000 \\
0.160 & 0.356 & 0.297 & 0.243 & 0.194 & 0.149 & 0.108 & 0.069 & 0.034 & 0.000 \\
0.180 & 0.367 & 0.307 & 0.252 & 0.202 & 0.156 & 0.113 & 0.073 & 0.036 & 0.000 \\
0.200 & 0.375 & 0.315 & 0.260 & 0.209 & 0.161 & 0.117 & 0.076 & 0.037 & 0.000 \\
0.220 & 0.381 & 0.321 & 0.265 & 0.214 & 0.166 & 0.121 & 0.078 & 0.038 & 0.000 \\
0.240 & 0.386 & 0.326 & 0.270 & 0.218 & 0.169 & 0.123 & 0.080 & 0.039 & 0.000 \\
0.260 & 0.390 & 0.330 & 0.274 & 0.221 & 0.172 & 0.125 & 0.082 & 0.040 & 0.000 \\
0.280 & 0.393 & 0.333 & 0.277 & 0.224 & 0.174 & 0.127 & 0.083 & 0.040 & 0.000 \\
0.300 & 0.396 & 0.335 & 0.279 & 0.226 & 0.176 & 0.128 & 0.084 & 0.041 & 0.000 \\
0.350 & 0.400 & 0.340 & 0.283 & 0.229 & 0.179 & 0.131 & 0.085 & 0.042 & 0.000 \\
0.400 & 0.402 & 0.342 & 0.285 & 0.231 & 0.180 & 0.132 & 0.086 & 0.042 & 0.000 \\
0.450 & 0.404 & 0.343 & 0.286 & 0.232 & 0.181 & 0.133 & 0.086 & 0.042 & 0.000 \\
0.500 & 0.405 & 0.344 & 0.287 & 0.233 & 0.182 & 0.133 & 0.087 & 0.042 & 0.000 \\
0.600 & 0.405 & 0.345 & 0.287 & 0.233 & 0.182 & 0.133 & 0.087 & 0.043 & 0.000 \\
0.800 & 0.405 & 0.345 & 0.288 & 0.234 & 0.182 & 0.134 & 0.087 & 0.043 & 0.000 \\
\hline
\end{tabular}

Table 3: Dimensionless Pressure Distribution for $r_{e D}=10, n=4$ and $\Delta t=0.05$

\begin{tabular}{cccccccccc}
\hline rD & & & & & & & & \\
tD & 1.0000 & 2.1250 & 3.2500 & 4.3750 & 5.5000 & 6.6250 & 7.7500 & 8.8750 & 10.0000 \\
\hline 10 & 1.639 & 0.923 & 0.547 & 0.327 & 0.191 & 0.107 & 0.055 & 0.023 & 0.000 \\
12 & 1.719 & 0.999 & 0.615 & 0.384 & 0.235 & 0.139 & 0.076 & 0.032 & 0.000 \\
14 & 1.719 & 0.999 & 0.615 & 0.384 & 0.235 & 0.139 & 0.076 & 0.032 & 0.000 \\
16 & 1.845 & 1.120 & 0.725 & 0.479 & 0.311 & 0.195 & 0.112 & 0.050 & 0.000 \\
18 & 1.896 & 1.170 & 0.770 & 0.518 & 0.343 & 0.219 & 0.128 & 0.058 & 0.000 \\
20 & 1.941 & 1.213 & 0.811 & 0.553 & 0.372 & 0.241 & 0.142 & 0.065 & 0.000 \\
25 & 2.032 & 1.302 & 0.892 & 0.624 & 0.430 & 0.285 & 0.171 & 0.079 & 0.000 \\
30 & 2.099 & 1.367 & 0.952 & 0.676 & 0.474 & 0.318 & 0.193 & 0.089 & 0.000 \\
35 & 2.149 & 1.415 & 0.996 & 0.715 & 0.506 & 0.342 & 0.209 & 0.097 & 0.000 \\
40 & 2.186 & 1.451 & 1.029 & 0.744 & 0.530 & 0.360 & 0.221 & 0.103 & 0.000 \\
45 & 2.213 & 1.477 & 1.054 & 0.766 & 0.547 & 0.374 & 0.230 & 0.107 & 0.000 \\
50 & 2.234 & 1.497 & 1.072 & 0.782 & 0.560 & 0.383 & 0.236 & 0.110 & 0.000 \\
55 & 2.249 & 1.511 & 1.085 & 0.793 & 0.570 & 0.391 & 0.241 & 0.113 & 0.000 \\
60 & 2.260 & 1.522 & 1.095 & 0.802 & 0.577 & 0.396 & 0.245 & 0.114 & 0.000 \\
65 & 2.268 & 1.530 & 1.103 & 0.809 & 0.583 & 0.400 & 0.247 & 0.116 & 0.000 \\
70 & 2.274 & 1.536 & 1.108 & 0.813 & 0.587 & 0.403 & 0.249 & 0.117 & 0.000
\end{tabular}




\begin{tabular}{cccccccccc}
\hline rD & 1.0000 & 2.1250 & 3.2500 & 4.3750 & 5.5000 & 6.6250 & 7.7500 & 8.8750 & 10.0000 \\
\hline 80 & 2.282 & 1.544 & 1.115 & 0.819 & 0.592 & 0.407 & 0.252 & 0.118 & 0.000 \\
90 & 2.286 & 1.548 & 1.119 & 0.823 & 0.594 & 0.409 & 0.253 & 0.119 & 0.000 \\
100 & 2.289 & 1.550 & 1.121 & 0.825 & 0.596 & 0.410 & 0.254 & 0.119 & 0.000 \\
110 & 2.290 & 1.552 & 1.122 & 0.826 & 0.597 & 0.411 & 0.254 & 0.119 & 0.000 \\
120 & 2.291 & 1.552 & 1.123 & 0.826 & 0.597 & 0.411 & 0.255 & 0.119 & 0.000 \\
130 & 2.291 & 1.553 & 1.123 & 0.827 & 0.598 & 0.412 & 0.255 & 0.119 & 0.000 \\
140 & 2.291 & 1.553 & 1.123 & 0.827 & 0.598 & 0.412 & 0.255 & 0.119 & 0.000 \\
160 & 2.292 & 1.553 & 1.124 & 0.827 & 0.598 & 0.412 & 0.255 & 0.119 & 0.000 \\
\hline
\end{tabular}

The results presented in Fig. 1 are the dimensionless pressure at the wellbore at different dimensionless time for the case of constant pressure outer boundary condition. When a reservoir is opened for production, a pressure disturbance is created in the reservoir from the wellbore. This disturbance is not only felt at the wellbore but it travels through the entire reservoir formation to the external boundary. Therefore, Tables 2 and 3 shows the dimensionless pressure at different points within and outside the wellbore of the reservoir against their corresponding dimensionless time.

\section{CONCLUSION}

This paper has been able to present the pressure distribution across a bounded circular reservoir assumed to have constant terminal rate at the wellbore. The diffusivity equation was used to analyse the pressure in the system. It was shown from Figs. 1 that the dimensionless pressure increases drastically immediately this flow regime is attained. But as time increases, the dimensionless pressure variation flattens out asymptotically. The results obtained from this analysis showed that there was a strong correlation with the results obtained from the Van Everdigen and Hurst. It is important to note that the Van Everdigen and Hurst solutions only state the pressure at the wellbore at a particular time but this work predicts the pressure variation in the entire reservoir from the wellbore to the external boundary at the same time. These where shown in Tables 2 and 3 and it was noticed that the pressure decreases from the wellbore to the external boundary of the reservoir. Therefore the Finite element method has been used to approximate not only the values of the wellbore pressures for bounded circular reservoirs but also pressure outside the wellbore to the external reservoir boundaries.

\section{REFERENCES}

[1] Razminia K., Hashemi A., and Razminia A. A Least Squares Approach to Estimating the Average Reservoir Pressure. Iranian J. Oil \& Gas Sci. Technol. 2(1), 22-32. 2013.

[2] Van Everdingen, A. F. and Hurst, W. The Application of the Laplace Transformation to Flow Problems in Reservoir. Trans., AIME 186, 305-324.1949.

[3] Essa K. S. M., Mina A. N., and Higazy M. Analytical Solution of Diffusion Equation in Two Dimensions Using Two Forms of Eddy Diffusivities. Rom. J. Phys. 56, 1228-1240. 2011.

[4] Oane M., Medianu R., Georgescu G., ToaderD., and Peled A. The determination of two photon thermal fields in laser-two-layer solids weak interactions using Green function method. Rom. Rep. Phys. 65, 997-1005. 2013.

[5] Timofte C. Homogenization Results for HyperbolicParabolic Equations, Rom. Rep. Phys. 62, 229-238. 2010.

[6] Earlougher, R.C., Jr., Ramey, H.J., Jr., Miller, F.G., and Mueller, T. D. Pressure Distributions in Rectangular Reservoirs. J. Pet. Tech., 199-208. 1968.

[7] Momoniat E., McIntyre R. and Ravindran R. Numerical inversion of a Laplace transform solution of a diffusion equation with a mixed derivative term. Appl. Math. Comput. 209(2), 222-229. 2009.

[8] Miller, C.C., Dyes, A.B., and Hutchinson, C.A., Jr. The Estimation of Permeability and Reservoir Pressure from Bottom-Hole Pressure Build-Up Characteristics. Trans., AIME189, 91-104. 1950.

[9] Aziz, K. and Flock, D. L. Unsteady State Gas Flow Use of Drawdown Data in the Rediction of Gas Well Behaviour. J. Can. Pet. Tech., 2 (1), 9-15. 1963.

[10] Earlougher R. C. Jr. Advances in Well Test Analysis, Monogragh Series, SPE, Dallas. 1977. 
[11] Ramey, H.J., Jr. and Cobb, W. M. A General Pressure Build-up Theory for a Well in a Closed Drainage Area. J. Pet. Tech., 1493 -1505. 1971.

[12] Kumar, A. and Ramey, H. J., Jr. Well-Test Analysis for a Well in a Constant-Pressure Square. Soc. Pet. Eng. J., 107-116. 1974.

[13] Cobb, W.M. and Smith, J. T. An Investigation of Pressure Build-up Tests in Bounded Reservoirs," J. Pet. Tech. Vol 27, No. 8, 1975, pp. 991 - 997.

[14] Chen, H.K. and Brigham, W. E. Pressure Build-up for a Well with Storage and Skin in a Closed Square. J. Pet. Tech., 141-146. 1978.

[15] Chatas, A. T. A Practical Treatment of Non-steadystate Flow Problems in Reservoir Systems. J. Pet. Eng., B-44-56. 1953.

[16] John L. Well Testing, Soc. Pet. Eng. of AIME, New York. 1982.
[17] Mishra, S. and Ramey, H. J., Jr. A New Derivative Type-Curve for Pressure Build-up Analysis with Boundary Effects. Proc., 12th Workshop on Geothermal Reservoir Engineering at Stanford Univ., Stanford, CA, 45-47. 1987.

[18] Ambastha, K., Anil and Henry J. Ramey, Jr. Well-Test Analysis for a Well in a Finite, Circular Reservoir. Proc., 13 ${ }^{\text {th }}$ Workshop on Geothermal Reservoir Engineering at Stanford Univ., Stanford, Califonia, 53-57. 1988.

[19] Agarwal, R. G. A New Method to Account for Producing Time Effects when Drawdown Type Curves are Used to Analyse Pressure Build-up and Other Test Data, paper SPE 9289 presented at the 55th Annual Meeting of SPE of AIME in Dallas, TX, 21-24. 1980. 